\title{
A Simple and Robust Cell-Based Assay for the Discovery of Novel Cytokinesis Inhibitors
}

Laszlo Radnai ${ }^{1,2}$, Rebecca F. Stremel ${ }^{1,2}$, Thomas Vaissiere ${ }^{2}$, Li Lin ${ }^{1}$, Michael Cameron ${ }^{1}$, William

H. Martin ${ }^{3}$, Gavin Rumbaugh ${ }^{2}$, Theodore M. Kamenecka ${ }^{1}$, Patrick R. Griffin ${ }^{1}$, Courtney A. Miller $^{1,2}$

${ }^{1}$ Department of Molecular Medicine, The Scripps Research Institute

${ }^{2}$ Department of Neuroscience, The Scripps Research Institute

${ }^{3}$ WHM Consulting, LLC, 111 Sterling City Rd., Lyme, CT 06371

Corresponding Author:

Courtney A. Miller, Departments of Molecular Medicine and Neuroscience, The Scripps

Research Institute, 130 Scripps Way, Jupiter, FL 33458, USA.

Email: CMiller@ scripps.edu

\section{Contents:}

\section{Figure S1}

2. Figure S2

\section{Figure S3}




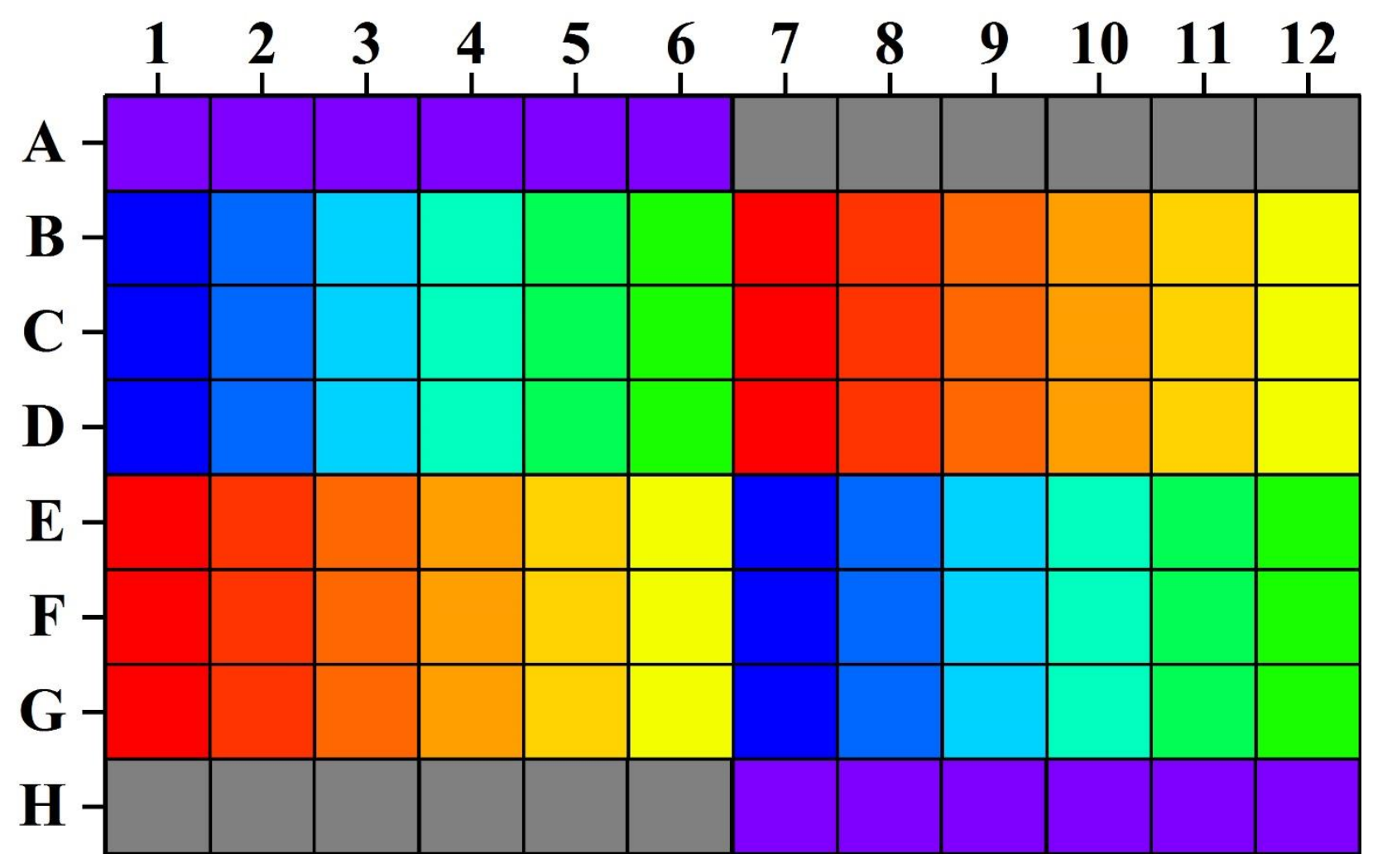

Figure S1. Assay plate layout used in screening experiments. Row A and row $\mathrm{H}$ were reserved for negative (1\% DMSO) and positive (20 $\mu \mathrm{M}$ para-aminoblebbistatin, 1\% DMSO) controls (violet and gray, respectively). Six-step serial 1:2 dilutions of four different compounds were prepared in DMSO and diluted in culture media. Subsequently, diluted compound solutions were transferred to the assay plate, such that every experiment was performed in triplicate. Blue-to-green and red-to-yellow color gradients (wells 1-6 and 7-12, rows B-D and E-F) represent the applied concentration gradients. 


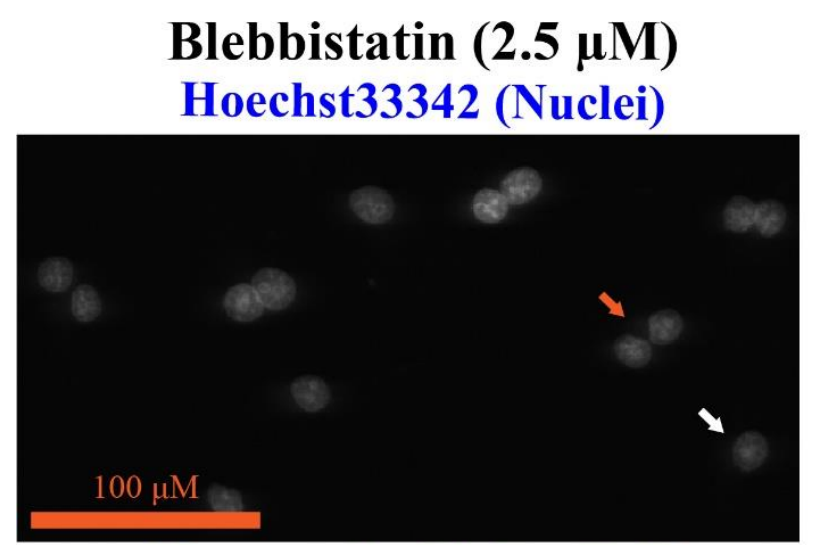

Fluorescein (Living Cells)

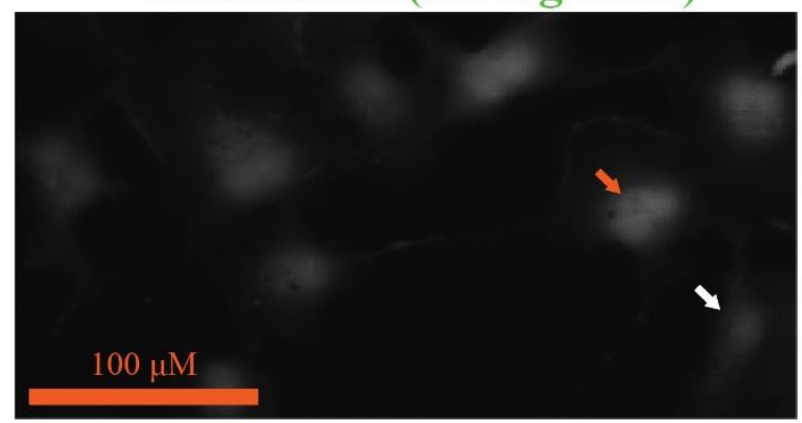

Overlay

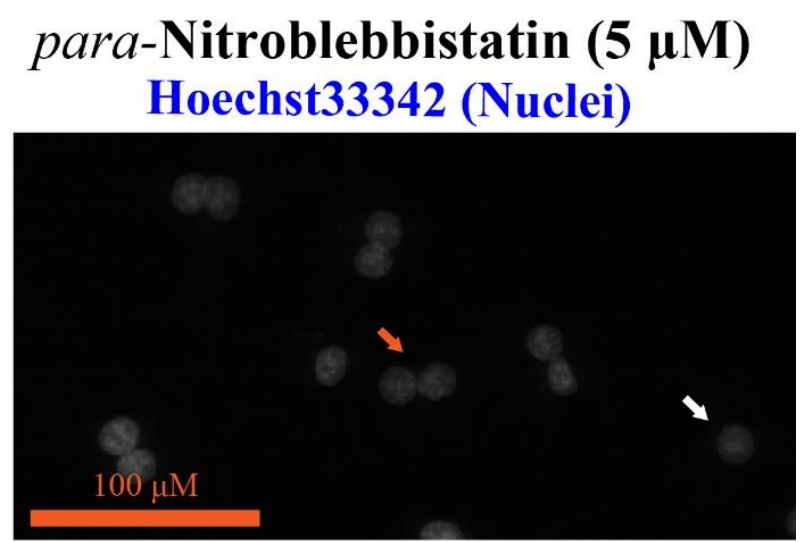

Fluorescein (Living Cells)

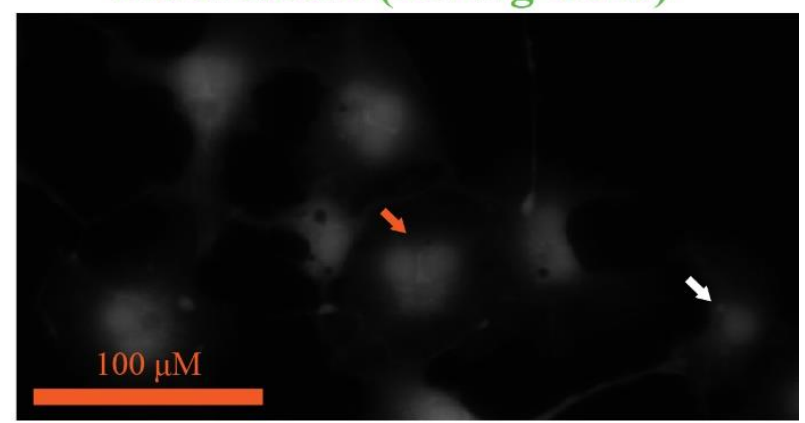

Overlay

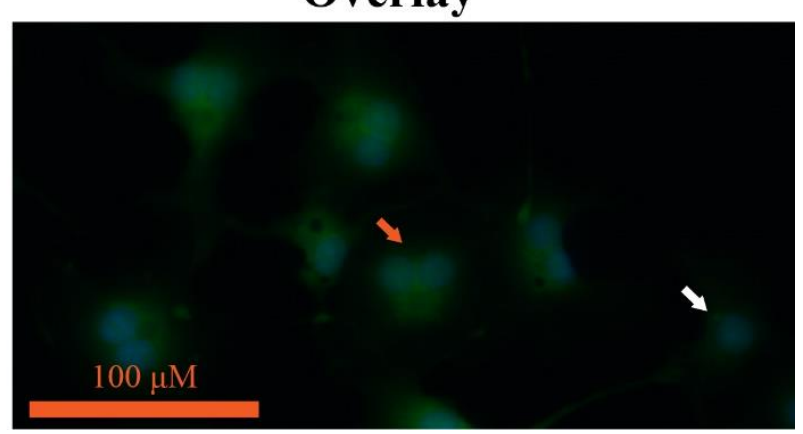

Figure S2. Cells treated with the NMII inhibitors blebbistatin (left) and para-nitroblebbistatin (right) develop multinucleated phenotype. Compounds were applied at the concentrations shown in parentheses and the cells were photographed after 24 hours of incubation. Images of nuclei (upper panels) and living cells (middle panels) were chosen to include both mono- (white arrows) and binucleated (orange arrows) cells. Overlaid images are shown to guide the eyes (lower panels, cytoplasm - green, nuclei - blue). Images were not manipulated other than changing the brightness 
and contrast for better visibility. No changes were made to the images prior to analysis. PI-positive (dead) nuclei were not observed in these fields of view. 
Jasplakinolide (31 nM)
Hoechst33342 (Nuclei)

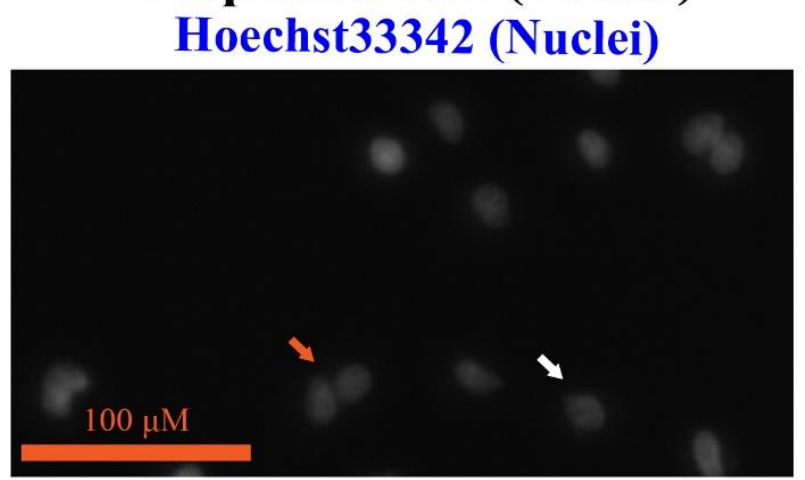

Fluorescein (Living Cells)

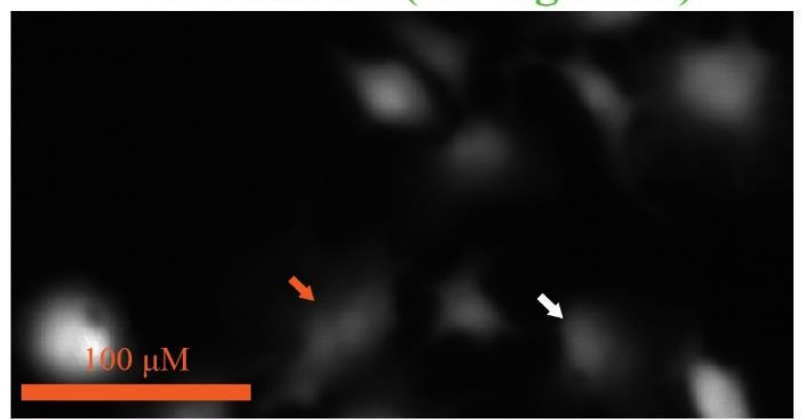

Overlay

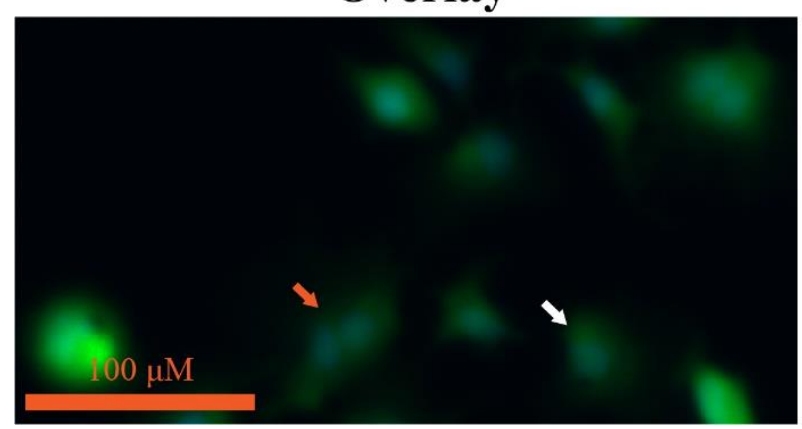

Swinholide A (2.5 nM)

Hoechst33342 (Nuclei)

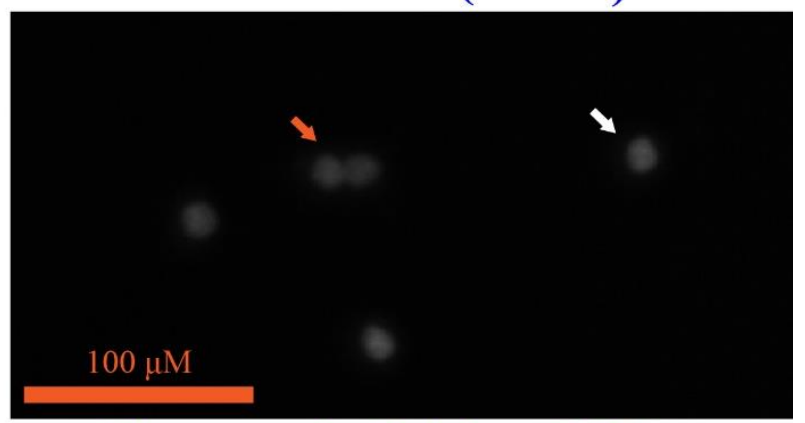

Fluorescein (Living Cells)

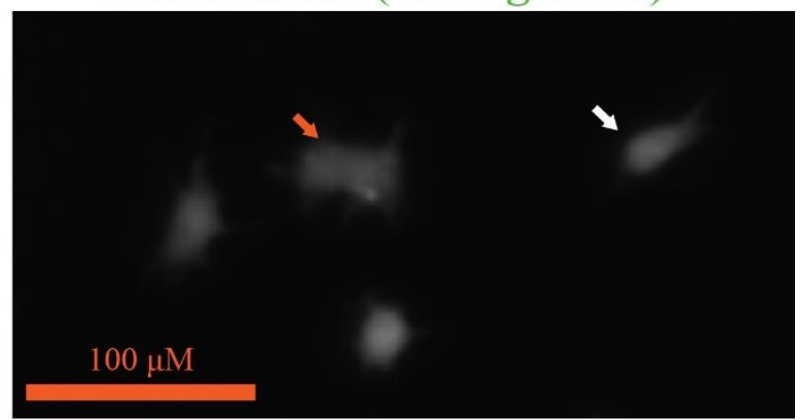

Overlay

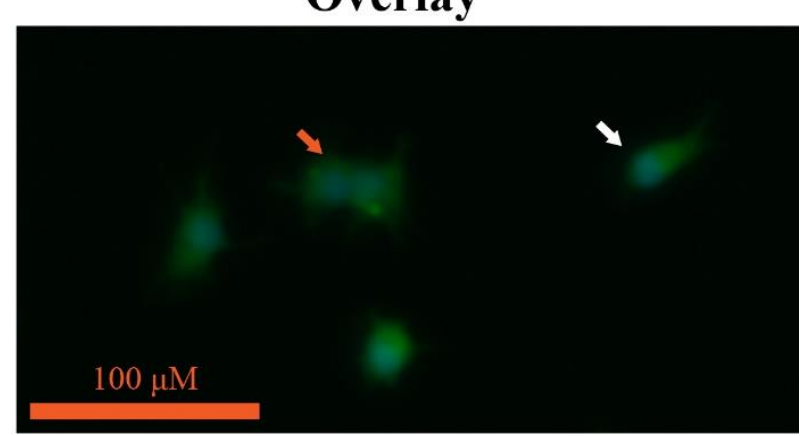

Figure S3. Cells treated with jasplakinolide (left), an inducer of actin polymerization, or swinholide A (right), an actin severing agent, develop a multinucleated phenotype. Compounds were applied at the concentrations shown in parentheses and the cells were photographed after 24 hours of incubation. Images of nuclei (upper panels) and living cells (middle panels) were chosen to include both mono- (white arrows) and binucleated (orange arrows) cells. Overlaid images are shown to guide the eyes (lower panels, cytoplasm - green, nuclei - blue). Images were not manipulated other than changing the brightness and contrast for better visibility. No changes 
were made to the images prior to analysis. PI-positive (dead) nuclei were not observed in these fields of view. 